\title{
Erratum: On the supersymmetric solutions of the Heterotic Superstring effective action
}

\author{
Andrea Fontanella $a^{a, b}$ and Tomás Ortín ${ }^{b}$ \\ ${ }^{a}$ Institut für Physik, Humboldt-Universität zu Berlin, \\ IRIS Gebäude, Zum Grossen Windkanal 6, 12489 Berlin, Germany \\ ${ }^{b}$ Instituto de Física Teórica UAM/CSIC, \\ C/Nicolás Cabrera, 13-15, C.U. Cantoblanco, E-28049 Madrid, Spain \\ E-mail: andrea.fontanella@physik.hu-berlin.de, tomas.ortin@csic.es
}

ERRATUM TO: JHEP06(2020)106

ARXIV EPRINT: 1910.08496

As pointed out in ref. [1], our paper contains an important error and a minor typo, both of which do not change the main conclusions. The error, though, is relevant because it affects one of the conditions a field configuration must satisfy in order to be supersymmetric.

Eq. (3.16) is wrong and, as a result, eq. (3.17) is also wrong and, once one takes into account that, in the coordinates and frame we have chosen, $\omega_{-m n}=-\omega_{m n-}$, it should be replaced by

$$
\Omega^{(+)}{ }_{-m n}=H_{-m n} .
$$

Then, eq. (3.18) does not follow immediately, although, in the end, it holds true: using the above result in the $a=-$ component of eq. (3.14a), we get

$$
\partial_{-} \Omega_{m_{1} m_{2} m_{3} m_{4}}=H_{-m_{1}}{ }^{n} \Omega_{n m_{2} m_{3} m_{4}},
$$

and, using the same properties as in the case of the $a=+$ component (eqs. (3.23) and (3.24)), we get

$$
H_{-m n}^{(-)}=\frac{1}{24} \Omega_{m}{ }^{s_{1} s_{2} s_{3}} \partial_{-} \Omega_{n s_{1} s_{2} s_{3}} .
$$

Then, eq. (3.31b) proves (3.18).

Since eqs. (3.16) and (3.17) are reproduced as eqs. (3.41a) and (3.41b), taking into account other results, these two equations should be replaced by

$$
\begin{aligned}
& \Omega_{[a b]-}^{(+)}=0, \\
& \Omega^{(+)}{ }_{a m-}=0, \\
& \Omega^{(+)}{ }_{-m n}=H_{-m n}^{(+)} .
\end{aligned}
$$


Accordingly, in the paragraph after eq. (3.27), the sentence "Via eqs. (3.16) they also determine the $H_{a b-}$ components of the Kalb-Ramond field strength." should be amended and replaced by "Via eqs. (3.15) they also determine the $H_{a m-}$ components of the KalbRamond field strength."

Above eq. (3.47) it should be said that we use the supersymmetry conditions eqs. (3.13) and (3.15) and the fact that, in the basis we are using, $\Omega^{(+)}{ }_{++-}=\omega_{++-}=0$ in order to simplify the gravitino KSE and reduce it to eq. (3.47).

Furthermore, eq. (3.17) was used to solve the - gravitino KSE. Using the old eq. (3.17) one would get eq. (3.49). Indeed, one gets that equation, but only after using the property (A.46a) and the projection eq. (3.44b):

$$
\left(\partial_{-}-\frac{1}{4} H^{(+)}{ }_{-m n} \Gamma^{m n}\right) \epsilon=\partial_{-} \epsilon=0 .
$$

Finally, there is small typo in eq. (3.47): the term $\partial_{m} \phi$ should be replaced by $\Gamma^{m} \partial_{m} \phi$.

Open Access. This article is distributed under the terms of the Creative Commons Attribution License (CC-BY 4.0), which permits any use, distribution and reproduction in any medium, provided the original author(s) and source are credited.

\section{References}

[1] P.A. Cano, Á. Murcia, P.F. Ramírez and A. Ruipérez, On small black holes, KK monopoles and solitonic 5-branes, JHEP 05 (2021) 272 [arXiv:2102.04476] [INSPIRE]. 